\title{
116. Note sur l'inégalité différentielle concernant les équations du type parabolique.
}

\author{
Par Mitio Nagumo et Seturo Simoda. \\ (Comm. by K. KUnUGI, M.J.A., Nov. 12, 1951.)
}

Un travail de valeur de H. WESTPHAL concernant les équations non-linéaires du type parabolique, nous avons vu dans un périodique allemand", que nous est arrivé tout récemment, en nous rappelant notre mémoire ancien ${ }^{2)}$, dans lequel nous mentionnâmes les pareilles matières aux siennes, et même fîmes l'amplification au cas plus général. Westphal a traité le théorème de comparaison, l'évaluation de solutions approximatives au moyen de la fonction majorante, l'unicité de solution sous la condition de Lipschitz et sous celle qui est plus générale, et a essayé d'une explication d'un phénomène très intéressant. On verra plus loin que l'inégalité différentielle (le théorème de comparaison) en question est dilatable à la forme quelque peu plus générale, de sorte qu'elle soit applicable aux équations non-linéaires du type parabolique et elliptique même.

1. Avant d'entrer en matière, nous allons énoncer quelques notions nécessaires dans la suite.

Étant donnée une fonction $\varphi(x, t, u, p, q, r)$ où $x=\left(x_{1}, \ldots, x_{m}\right)$, $p=\left(p_{1}, \ldots, p_{m}\right)$ et $r=\left(r_{i j}: i, j=1, \ldots, m\right)$, définie dans une région $R$ dans l'espace $E^{2 m+3+m} 2$ s) jouissant de la symétrie telle que $\Phi$ reste invariante sous la substitution de $r_{i j}$ par $r_{j i}(i, j=1, \ldots, m)$, nous entendons par fonction semi-elliptiqne en $x$ relative $\grave{a} \phi$ telle fonction $u(x, t)$, définie et dérivable en $t$ et de deux fois en $x$ dans une région située dans $E^{m+1}$, que tous les points $\left(x, t, u(x, t), \partial_{x} u(x, t)\right.$, $\left.\partial_{t} u(x, t), \partial_{x}^{2} u(x, t)\right)^{4)}$ appartiennent à $R$, et qu'il existe, pour tout $(x, t, r)$ tel que $\left(x, t, u(x, t), \partial_{x} u(x, t), \partial_{t} u(x, t), r\right) \in R$, les nombres $\Phi_{i j}=\Phi_{j i}(i, j=1, \ldots, m)$ dépendants de $(x, t, r)$, de sorte qu'on ait

1) H, Westphal : Zur Abschätzung der Lösungen nicht linearer parabolischer Differentialgleichungen. Math. Zeits. 51 (1949), pp. 690-695.

2) La note de Nagumo dans “Kansū-Höteisiki" No. 15 (1939), en japonais.

$3) E^{n}$ désigne l'éspace euclidien à $n$-dimension.

4) Pour simplicité nous osons écrire $\partial_{i} u(x, t)$ pour $\frac{\partial}{\partial x_{i}} u(x, t), \partial_{t} u(x, t)$ pour $\frac{\partial}{\partial t} u(x, t), \partial_{i j}^{2} u(x, t)$ pour $\frac{\partial^{2}}{\partial x_{i} \partial x_{j}} u(x, t)$, et nous servons des abréviations $\partial_{x} u$, $\left.=\partial_{1} u, \ldots, \partial_{m} u\right), \partial_{x}^{2} u=\left(\partial_{i j}^{2} u: i, j=1, \ldots, m\right)$. 


$$
\left\{\begin{array}{l}
\sum_{i, j=1}^{m} \Phi_{i j} \lambda_{i} \lambda_{j} \geq 0 \quad \text { pour tous réels } \lambda_{k}(k=1, \ldots, m), \\
\Phi\left(x, t, u, \partial_{x} u, \partial_{t} u, r\right)-\Phi\left(x, t, u, \partial_{x} u, \partial_{t} u, \partial_{x}^{2} u\right) \\
\quad=\sum_{i, j=1}^{m} \Phi_{i j}\left\{r_{i,}-\partial_{i j}^{2} u(x, t)\right\}^{n)} .
\end{array}\right.
$$

Ensuite, dans la considération d'un ensemble ouvert $D$ de point $(x, t)=\left(x_{1}, \ldots, x_{m}, t\right)$ dans $E^{m+1}$, nous désignons par $S$ une partie de la frontière de $D$, la partie qui ne consiste qu'en point $(x, t)$ tel que, avec quelque $\varepsilon>0$, les inégalitís $|x-\xi|<\varepsilon^{6)}$ et $0 \leqq t-\tau<\varepsilon$ entraînent $(\xi, \tau) \in D \cup S^{7}$. (Cf. Fig. 1.)

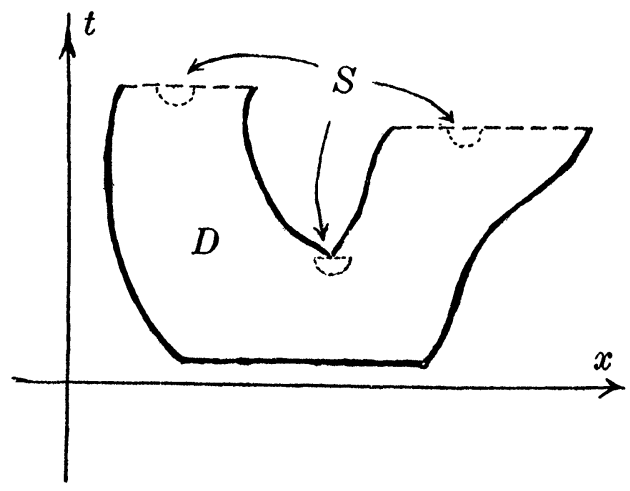

Fig. 1.

ThÉoRème DE Comparaison: Soient $v(x, t)$ et $w(x, t)$ des fonctions définies dans $D \cup S$, jouissant des propriétés suivantes:

$1^{\circ}$ Elles sont. dans $D \cup S$, continues en $(x, t)$, continûment dérivables en $x$ de deux fois, et dérivables en $t$.

$2^{\circ}$ Pour toute suite $\left\{\left(x^{(n)}, t^{(n)}\right): n=1,2,3, \ldots \ldots\right\}$ dans $D \cup S$ n'ayant pas de point d'accumulation dans là, on a toujours

$$
\varliminf_{n \rightarrow \infty}\left\{w\left(x^{(n)}, t^{(n)}\right)-v\left(x^{(n)}, t^{(n)}\right)\right\}>0 .
$$

De plus nous supposons les suivants :

$3^{\circ} \varphi(x, t, u, p, q, r)$ est la fonction écrite plus haut et noncroissant en $q$; à dire avec détail, dès qu'on aura $(x, t, u, p, q, r)$

5) Des cas où se prêtre $\emptyset$ à cette condition, sont les suivants :

(i) $\emptyset=\Phi\left(x, t, u, p, q, r_{11}, r_{29}, \ldots, r_{m m}\right)$, c'est-à-dire, $\emptyset$ est affranchie de toutes $r_{i j}(i \neq j)$, en outre $\emptyset$ est non-croissante en chaque $r_{i i}(i=1, \ldots, m)$.

(ii) $\emptyset$ est continûment dérivable par papport à $r$ et la forme quadratique $\sum_{i, j}^{m} \partial_{r_{i j}} \Phi \lambda_{i} \lambda_{j}$ est semi-définie positive, toute section de $R$ par l'hyperplan parallèle à l'espace de $r$ étant supposée ouverte et convexe.

6) $|x-\xi|$ désigne la distance de deux points $x, \xi$.

7) Référez au suivant: Mauro Picone: Maggiorazione degli integrali delle equazioni totalemente paraboliche alle derivate parziali del secondo ordine. Ann. di Math. p. ed a., IV, 7 (1929), pp. 145-192, spécialement p. 147 et p. 180. 
$\epsilon R$, tous $\left(x, t, u, p, q^{\prime}, r\right)$ avec $q^{\prime} \geqq q$ aussi appartiendront à $R$ et on aura

$$
\Phi(x, t, u, p, q, r) \geqq \Phi\left(x, t, u, p, q^{\prime}, r\right) .
$$

$4^{\circ}$ Tous les points $\left(x, t, w(x, t), \partial_{x} w(x, t), \partial_{t} w(x, t), \partial_{x}^{2} w(x, t)\right)$ avec $(x, t) \in D \cup S$ appartiennent à $R$ tandis que $v(x, t)$ est semielliptique en $x$ relative $\grave{a} \Phi$, et on a toujours

$$
\Phi\left(x, t, v, \partial_{x} v, \partial_{t} v, \partial_{x}^{2} v\right)>\Phi\left(x, t, w, \partial_{x} w, \partial_{t} w, \partial_{x}^{2} w\right)
$$

\section{Alors l'inégalité}

$$
v(t, x)<w(t, x)
$$

subsiste pour tous $(x, t) \in D \cup S$.

REMARQUE 1: En réalité, il suffit, pour notre conclusion, qu'on a l'inégalité (1) seulement pour tout $(x, t) \in D \cup S$ tel que les relations

et

$$
\begin{aligned}
v(x, t)= & w(x, t), \partial_{x} v(x, t)=\partial_{x} w(x, t) \\
& \partial_{t} v(x, t) \geqq \partial_{t} w(x, t)
\end{aligned}
$$

ont lieu simultanément.

2. Preuve du théorème: Posons

$$
\begin{aligned}
& F=\{(x, t): \quad w(x, t) \leqq v(x, t)\} \\
& F_{t}=\{t: \quad(x, t) \in F \text { avec quelque } x\} .
\end{aligned}
$$

$F$ est un sous-ensemble fermé de $D \cup S$ et $F_{t}$ la projection orthogonale de $F^{\prime}$ sur l'axe de $t$. Nous supposons qu'ils ne soient pas vides.

$F_{t}$ est alors inférieurement borné, et $\bar{F}$ contenue dans $D \cup S$ (partant $\bar{F}=F)$, car sinon il existe une suite $\left\{\left(x^{(n)}, t^{(n)}\right): n=1,2\right.$, $3, \ldots \ldots\}$ dans $F$ n'ayant pas de point d'accumulation dans $D \cup S$ et elle donne

$$
\varlimsup_{n \rightarrow \infty}\left\{w\left(x^{(n)}, t^{(n)}\right)-v\left(x^{(n)}, t^{(n)}\right)\right\} \leqq 0,
$$

au contraire de l'hypothèse $2^{\circ}$. On peut donc prendre un point $\left(x_{0}, t_{0}\right) \in F$ tel que $t_{0} \leqq t$ pour tout $(x, t) \in F$.

Or on pourrait aisément voir que la fonction (en $x) w\left(x, t_{0}\right)-$ $v\left(x, t_{0}\right)$ est $\geqq 0$ pour tout $\left(x, t_{0}\right) \in D \cup S$ vu la continuité en $t$ des $v$ et $w$, atteignant la valeur 0 à $\left(x_{0}, t_{0}\right)$; cela étant son minimum, il en résulte que

$$
\left\{\begin{array}{c}
w\left(x_{0}, t_{0}\right)=v\left(x_{0}, t_{0}\right) \\
\partial_{i} w\left(x_{0}, t_{0}\right)=\partial_{i} v\left(x_{0}, t_{0}\right)(i=1, \ldots, m),
\end{array}\right.
$$


et que la matrice $\left(\partial_{i j}^{2} w\left(x_{0}, t_{0}\right)-\partial_{i j}^{2} v\left(x_{j}, t_{0}\right): i, j=1, \ldots, m\right)$ est semidéfinie positive, en outre que

$$
\partial_{t} w\left(x_{0}, t_{0}\right) \leqq \partial_{t} v\left(x_{0}, t_{0}\right)
$$

d'après la définition de $\left(x_{0}, t_{0}\right)$. Donc on a

$$
\begin{aligned}
\Delta & =\Phi\left(x_{0}, t_{0}, w_{0}, \partial_{x} w_{0}, \partial_{t} w_{0}, \partial_{x}^{2} w_{0}\right)^{8)} \\
& -\Phi\left(x_{0}, t_{0}, v_{0}, \partial_{x} v_{0}, \partial_{t} v_{0}, \partial_{x}^{2} v_{0}\right) \\
& =\Phi\left(x_{0}, t_{0}, w_{0}, \partial_{x} w_{0}, \partial_{t} w_{0}, \partial_{x}^{2} w_{0}\right) \\
& -\Phi\left(x_{0}, t_{0}, w_{0}, \partial_{x} w_{0}, \partial_{t} v_{0}, \partial_{x}^{2} w_{0}\right) \\
& +\Phi\left(x_{0}, t_{0}, v_{0}, \partial_{x} v_{0}, \partial_{t} v_{0}, \partial_{x}^{2} w_{0}\right) \\
& -\Phi\left(x_{0}, t_{0}, v_{0}, \partial_{x} v_{0}, \partial_{t} v_{0}, \partial_{x}^{2} v_{0}\right) \\
\geqq & \sum_{i, j=1}^{m} \Phi_{i j}\left\{\partial_{i j}^{2} w\left(x_{0}, t_{0}\right)-\partial_{i j}^{2} v\left(x_{0}, t_{0}\right)\right\},
\end{aligned}
$$

vu les hypothèses $3^{\circ}, 4^{\circ}$ et les relations (2), (3); le dernier terme précédent étant la trace du produit multiplicatif des deux matrices symétriques, toutes lesquelles sont semi-définies positives, on a $\Delta \geqq 0^{9)}$ contrairement à l'hypothése $4^{\circ}$ (l'inégalité (1)). Cela donne $F=$ vide, aussi la conclusion voulue est claire.

Remarque 2: Le théorème reste vrai même au cas où $w(x, t)$ est semi-elliptique en $x$ relative à $\phi$, c'est-à-dire, où les $v$ et $w$ échangent des caractères, pourvu qu'on change de direction du signe de l'inégalité dans l'hypothèse $2^{\circ}$.

REMARQUe 3: Si $\Phi$ est affranchie de $q$, le théorème illumine, concernant l'equation du type elliptique, la immuabilité de l'inégalité contre la variation du paramètre $t$. Pour cela, voir notre mémoire précédent. ${ }^{10}$

Application : Envisageons les inégalitós

$$
\begin{aligned}
& \partial_{t} v \leqq f\left(x, t, v, \partial_{x} v, \partial_{x}^{2} u\right), \\
& \partial_{t} w>f\left(x, t, w, \partial_{x} w, \partial_{x}^{2} w\right),
\end{aligned}
$$

où $\phi(x, t, u, p, q, r)=f(x, t, u, p, r)-q$ satisfait à une des conditions écrites dans la note 5) au bas de la page 537. Si $v$ et $w$ remplissent les conditions du théorème, l'inégalité $v(x, t)<w(x, t)$ subsiste pour tout $(x, t) \in D \cup S$; c'est bien le cas qu'a traité Westphal pour $m=1$.

8) Le symbole o signifie qu'on pose $\left(x_{0}, t_{0}\right)$ pour $(x, t)$.

9) Voir : M. Picone, ibid. p. 178, le lemme de Moutard.

10) S. Simoda et M. Nagumo: Sur la solution bornée de l'équation aux dérivées partielles du type elliptique. Proc. Japan Acad., 27 (1951), No. 7, pp. 334-339. 